IP

\title{
Durabilidade natural das madeiras de Apuleia leiocarpa, Astronium lecointei e Enterolobium schomburgkii ao fungo apodrecedor Trametes versicolor
}

\author{
Magnos Alan Vivian ${ }^{1 *(D)}$, Karina Soares Modes ${ }^{1}$ (D), Adriana Terumi Itako1(D), Paola Cristine Pereira da Cruz ${ }^{1}$, Évelyn Janaina Grosskopf ${ }^{1}$ (D), \\ Gláucia Cota Nunes ${ }^{1}(\mathbb{D}$
}

${ }^{1}$ Universidade Federal de Santa Catarina, Rod. Ulysses Gaboardi, Km 3, CEP 89520-000, Curitibanos, SC, Brasil

*Autor correspondente:

magnos.alan@ufsc.br

Termos para indexação:

Resistência da madeira

Apodrecimento da madeira

Fungo xilófago

Index terms:

Wood resistance

Rotting wood

Xylophagous fungus

Histórico do artigo:

Recebido em 05/07/2018

Aceito em 05/12/2018

Publicado em 29/12/2018

doi: 10.4336/2018.pfb.38e201801664
Resumo - O presente estudo teve por objetivo avaliar a durabilidade natural das madeiras de Apuleia leiocarpa (grápia), Astronium lecointei (muiracatiara) e Enterolobium schomburgkii (fava-orelha-de-negro) ao ataque do fungo causador da podridão branca Trametes versicolor (Linnaeus ex Fries) Pilat, pelo ensaio de apodrecimento acelerado. Foram utilizadas 15 amostras de cerne de cada espécie, nas dimensões 2,5 x 2,5 x 0,9 cm (radial, tangencial e axial, respectivamente). Essas permaneceram durante 16 semanas em incubadora sob ataque do fungo. Após o período de ataque, determinaram-se a perda de massa e a referida classe de durabilidade natural. E. schomburgkii apresentou a maior média de perda de massa (17,5\%), seguida de A. lecointei $(15,5 \%)$, sendo classificadas como resistentes a $T$. versicolor. A. leiocarpa apresentou maior durabilidade $(10,6 \%$ de perda de massa), sendo classificada como altamente resistente. A madeira de $A$. leiocarpa diferiu estatisticamente das demais, indicando potencial de maior durabilidade quando disposta em condições que favoreçam a desenvolvimento deste xilófago.

\section{Natural durability of the woods of Apuleia leiocarpa, Astronium lecointei and Enterolobium schomburgkii to the rotting fungus Trametes versicolor}

\section{Introdução}

O Brasil possui a segunda maior cobertura florestal do mundo, com $12 \%$ da área global, seguido da Rússia, com $20 \%$ da área total (FAO, 2015). Por esse motivo,

\begin{abstract}
The objective of the present study was to evaluate the natural durability of the wood of Apuleia leiocarpa (grápia), Astronium lecointei (muiracatiara) and Enterolobium schomburgkii (fava-orelha-de-negro) under the attack of fungi causing white rot Trametes versicolor (Linnaeus ex Fries) Pilat, under controlled laboratory conditions, by the accelerated rotting test. We used 15 samples of heartwood of each species, with $2.5 \times 2.5 \times 0.9 \mathrm{~cm}$ (radial, tangential and axial, respectively). The samples remained for 16 weeks in an incubator under attack of the fungus. After the period of the attack the mass loss and class of natural durability were determined. E. schomburgkii presented the highest average loss of mass (17.5\%), followed by A. lecointei (15.5\%), both classified as resistant to $T$. versicolor. A. leiocarpa wood presented higher durability (10.6\% loss of mass), being classified as highly resistant. The wood of $A$. leiocarpa differed statistically from the others, indicating potential of greater durability when placed under condition that favor the development of this xylophagus.
\end{abstract}

o país apresenta características singulares de produção florestal, possuindo potencial que permite a oferta de produtos madeireiros e não madeireiros, tanto de espécies plantadas quanto nativas. 
Apesar da grande diversidade de espécies arbóreas nas florestas naturais brasileiras, estudos apontam que a maioria delas ainda tem seu uso potencial desconhecido. Essas podem ser utilizadas para a obtenção de produtos madeireiros e não madeireiros, constituindo uma importante fonte de geração de renda e empregos (Brasil, 2006).

$\mathrm{O}$ atendimento à demanda futura de madeira só será alcançado se houver um aumento na eficiência da produção, da exploração, da conversão da matériaprima e da promoção de novas espécies florestais, que atendam padrões de qualidade exigidos pelo mercado internacional (Brasil, 2006).

Em países tropicais, como o Brasil, um dos principais fatores para utilização da madeira é a sua durabilidade natural (Mendes \& Alves, 1988). Tal parâmetro fornece informações para planos de uso específico da madeira, gerando resultados que a classificam quanto ao uso ou não em contato com o solo, complementando o conhecimento das suas demais propriedades tecnológicas (Trevisan, 2008). A durabilidade natural da madeira pode ser entendida como sua capacidade de resistir à ação de agentes deterioradores, sejam eles bióticos ou abióticos. Os agentes bióticos de maior importância são os fungos, responsáveis pela maior proporção de danos causados à madeira, podendo comprometer severamente sua resistência (Morais et al., 2005).

Trametes versicolor é um dos mais eficientes fungos degradadores da madeira, que promove a deterioração simultânea da lignina, da celulose e da hemicelulose (Tanaka et al., 1999), causando a podridão branca (Archibald \& Addleman, 1997). Esta deterioração pode ser entendida com a alteração das propriedades da madeira, como perda de massa, mudança na coloração e redução da resistência mecânica.

Considerando-se que madeiras classificadas como resistentes à deterioração apresentam ampla aceitação e difusão de emprego no mercado madeireiro, o objetivo desse estudo foi avaliar a durabilidade natural das madeiras de Apuleia leiocarpa, Astronium lecointei e Enterolobium schomburgkii em ensaio de apodrecimento acelerado expostas ao fungo de podridão branca Trametes versicolor, em condições controladas de laboratório.

\section{Material e métodos}

Foram utilizadas madeiras de três espécies florestais nativas obtidas na xiloteca da Universidade Federal de
Santa Catarina, Campus de Curitibanos, sendo: Apuleia leiocarpa (grápia), Astronium lecointei (muiracatiara) e Enterolobium schomburgkii (fava-orelha-de-negro), procedentes de material cedido pelo Instituto Brasileiro do Meio Ambiente e dos Recursos Naturais Renováveis (IBAMA). A escolha dessas espécies deu-se em função da escassez de estudos com as mesmas bem como pelo fato de já terem apresentado em algum momento da história, importância quanto ao seu uso em produtos florestais madeireiros.

A susceptibilidade das espécies madeireiras ao ataque do fungo apodrecedor foi determinada por meio do ensaio de apodrecimento acelerado em laboratório, de acordo com a norma ASTM D 2017 (American Society for Testing and Materials, 2005). Os procedimentos foram conduzidos no Laboratório de Recursos Florestais e no Laboratório de Fitopatologia da mesma Universidade.

Confeccionou-se 15 corpos de prova para cada espécie, nas dimensões 2,5 x 2,5 x $0,9 \mathrm{~cm}$ (radial, tangencial e axial, respectivamente), todos retirados em região de cerne. Os corpos de prova foram lixados, visando uniformizar a superfície e posteriormente identificados.

Foi utilizado o fungo xilófago Trametes versicolor, espécie causadora da podridão branca, proveniente da coleção do Laboratório de Produtos Florestais (LPF), do Serviço Florestal Brasileiro (SFB), de Brasília, DF.

Para a preservação do fungo até o momento da execução do ensaio, foi utilizada a metodologia de Castellani (1964), que consiste em manter a cultura em água destilada em temperatura ambiente.

\section{Preparo do substrato e frascos do ensaio}

Foi utilizado solo coletado de horizonte B, livre de matéria orgânica e com pH próximo de 6,0. Este foi macerado e peneirado para eliminação de pedras e impurezas.

Foram adicionados $100 \mathrm{~g}$ de solo em frascos de vidro transparentes, com tampas rosqueáveis e capacidade de $600 \mathrm{~mL}$. Em seguida, acrescentou-se 36,3 g de água destilada a este substrato, a fim de ajustar a umidade do solo para $130 \%$ da sua capacidade de retenção de água, segundo as recomendações da ASTM D 1413 (American Society for Testing and Materials, 1999).

Cada frasco recebeu uma placa suporte de madeira medindo $0,3 \times 3,0 \times 3,0 \mathrm{~cm}$ (radial, tangencial e longitudinal), do gênero Eucalyptus, devido ao fungo T. versicolor apresentar bom desenvolvimento sobre espécies de folhosas. 
Em seguida, os frascos foram fechados e sobre cada tampa acrescentado papel alumínio, com o intuito de evitar contaminação. Posteriormente, foram autoclavados, a uma temperatura de aproximadamente $121{ }^{\circ} \mathrm{C}$ por $30 \mathrm{~min}$.

\section{Produção do inóculo}

Inicialmente o fungo $T$. versicolor foi cultivado em placas com meio de cultura BDA (Batata-DextroseÁgar), extrato composto de $200 \mathrm{~g}$ de batata, $20 \mathrm{~g}$ de dextrose, $20 \mathrm{~g}$ de ágar e $1000 \mathrm{~mL}$ de água destilada, autoclavado por 20 min em pressão de $1 \mathrm{~atm}$ e $121^{\circ} \mathrm{C}$.

A repicagem foi conduzida em capela de fluxo laminar horizontal devidamente esterilizada, em que um disco contendo micélios do fungo foi adicionado ao meio de cultura disposto no interior de placas de Petri. Após tal procedimento, estas foram mantidas em incubadora de crescimento a de $25 \pm 1{ }^{\circ} \mathrm{C}$, em fotoperíodo de $12 \mathrm{~h}$, por aproximadamente 15 dias, para que houvesse a completa colonização da placa pelo fungo.

A inoculação do fungo sobre a placa suporte foi efetuada em capela asséptica, com cinco discos de micélio com aproximadamente $10 \mathrm{~mm}$, provenientes das placas de Petri. Depois de inoculados, os frascos retornaram à incubadora onde permaneceram por um período de 15 dias, necessários para que o micélio do fungo cobrisse homogeneamente a superfície do substrato (placa suporte).

\section{Registro da massa inicial e esterilização dos corpos de prova}

Antes dos corpos de prova das espécies florestais serem submetidos ao ensaio de apodrecimento, estes passaram pelo processo de estabilização para obter sua massa inicial, após um período de secagem, em estufa a $60 \pm 1{ }^{\circ} \mathrm{C}$ por aproximadamente 20 dias. $\mathrm{O}$ monitoramento do peso do material foi conduzido em um grupo pequeno de amostras, até que estas atingissem sua estabilidade. Verificada a estabilização do lote de amostragem, todos os corpos de prova tiveram a massa inicial registrada. Para a pesagem foi utilizada balança analítica, com precisão de 0,0001 g. Os corpos de prova, antes da inoculação, foram esterilizados em autoclave a $110 \pm 10{ }^{\circ} \mathrm{C}$ por um período de $1 \mathrm{~h}$.

\section{Inserção das amostras e período de ensaio}

Para dar início ao ensaio de apodrecimento acelerado propriamente dito, um corpo de prova por espécie foi inserido no interior dos frascos sobre a placa suporte já colonizada pelo fungo.
Finalizado esse procedimento, os frascos retornaram à incubadora, a $25 \pm 1^{\circ} \mathrm{C}$ e fotoperíodo de $12 \mathrm{~h}$, onde permaneceram por um período de 16 semanas, conforme a norma utilizada. Durante esse período os frascos foram aleatoriamente trocados de lugar semanalmente, para que não houvesse nenhuma interferência do posicionamento no interior do equipamento.

O experimento foi finalizado o período na incubadora, os corpos de prova foram retirados dos frascos, e com o auxílio de uma pinça, os micélios aderidos aos mesmos foram cuidadosamente removidos, utilizando-se uma escova de cerdas macias.

A massa final foi registrada após processo de estabilização idêntico ao adotado para o registro da massa inicial. Assim, os corpos de prova deteriorados foram condicionados em estufa a $60 \pm 1{ }^{\circ} \mathrm{C}$, por aproximadamente 20 dias, até que atingissem massa constante, monitorada em balança analítica.

\section{Avaliação da perda de massa e classificação da durabilidade natural da madeira}

A perda de massa das madeiras, ocasionada pelo ataque do fungo apodrecedor, foi obtida a partir da Equação 1, e a massa residual, pela Equação 2.

$P M=\frac{M i-M}{M i} \times 100$

$M R=100-P M$

Em que: $\mathrm{PM}=$ perda de massa (\%); $\mathrm{Mi}=$ massa da amostra antes do ataque do fungo $(\mathrm{g}) ; \mathrm{Mf}=$ massa da amostra após o ataque do fungo $(\mathrm{g}) ; \mathrm{MR}=$ massa residual $(\%)$.

Em seguida, classificou-se a durabilidade natural das madeiras a partir da tabela de classes de resistência (Tabela 1).

Por fim, aplicou-se o teste de normalidade e homogeneidade dos dados, e a partir do resultado destes procedeu-se a análise de variância (ANOVA), para avaliar a perda de massa das três espécies, com posterior comparação de médias pelo teste de Tukey a $5 \%$ de probabilidade, através do programa Sisvar. 
Tabela 1. Classificação da durabilidade natural de madeiras submetidas ao ensaio de apodrecimento acelerado, segundo a norma ASTM D 2017 (American Society for Testing and Materials, 2005).

Table 1. Classification of the natural durability of wood subjected to the accelerated rotting test, according to ASTM D 2017 (American Society for Testing and Materials, 2005).

\begin{tabular}{ccc}
\hline Perda de massa (\%) & Massa residual (\%) & Classe de resistência \\
0 a 10 & 100 a 90 & Altamente resistente \\
11 a 24 & 89 a 76 & Resistente \\
25 a 44 & 76 a 56 & Resistência moderada \\
45 ou mais & 55 ou menos & Não resistente \\
\hline
\end{tabular}

\section{Resultados}

Após as 16 semanas de exposição das amostras de madeira ao fungo Trametes versicolor foram obtidos os valores de perda de massa e massa residual expressos na Tabela 2.

Tabela 2. Durabilidade natural das madeiras de Apuleia leiocarpa, Astronium lecointei e Enterolobium schomburgkii, ao fungo apodrecedor Trametes versicolor.

Table 2. Natural durability of Apuleia leiocarpa, Astronium lecointei and Enterolobium schomburgkii woods to rotting fungus Trametes versicolor.

\begin{tabular}{cccccccc}
\hline & \multicolumn{2}{c}{ A. leiocarpa } & \multicolumn{2}{c}{ A. lecointei } & \multicolumn{2}{c}{ E. schomburgkii } \\
\cline { 2 - 7 } & \multicolumn{2}{c}{ PM } & MR & PM & MR & PM & MR \\
\cline { 2 - 7 } & \multicolumn{2}{c}{$(\mathbf{\%})$} & \multicolumn{2}{c}{$(\mathbf{\%})$} & \multicolumn{2}{c}{$\mathbf{( \% )}$} \\
\hline Mínimo & 4,9 & 81,2 & 8,2 & 73,2 & 8,7 & 79,4 \\
Máximo & 18,8 & 95,1 & 26,8 & 91,8 & 20,6 & 91,3 \\
Médio & $10,6 \mathrm{a}$ & 89,4 & $15,5 \mathrm{~b}$ & 84,5 & $17,5 \mathrm{~b}$ & 82,5 \\
\hline CV\% & 36,8 & 4,4 & 32,2 & 5,9 & 18,5 & 3,9 \\
\hline \multirow{2}{*}{ CR } & \multicolumn{2}{c}{$\begin{array}{c}\text { Altamente } \\
\text { resistente }\end{array}$} & \multicolumn{2}{c}{ Resistente } & \multicolumn{2}{c}{ Resistente } \\
\hline
\end{tabular}

Em que: $\mathrm{PM}=$ perda de massa; $\mathrm{MR}=$ massa residual $; \mathrm{CV} \%=$ coeficiente de variação; $\mathrm{CR}=$ classe de resistência. Médias de PM que possuem a mesma letra não diferem estatisticamente entre si, de acordo com teste Tukey a $5 \%$ de probabilidade.

As madeiras de Astronium lecointei e Enterolobium schomburgkii, em função da perda de massa apresentada após período de exposição ao fungo de podridão branca utilizado, tiveram sua durabilidade natural classificada como resistente. A. leiocarpa foi classificada como altamente resistente, pois apresentou perda de massa inferior a $11 \%$.

\section{Discussão}

Estudos conduzidos pelo Ibama (1997) mostraram a espécie Astronium lecointei como muito durável. Ao avaliarem a durabilidade da madeira de Apuleia leiocarpa em ensaio de laboratório, Fosco Mucci et al. (1992) classificaram a mesma como resistente aos fungos apodrecedores Trametes versicolor, Gloeophyllum trabeum e Poria monticola. Esses resultados são consistentes ao com o presente trabalho (Tabela 2).

Em estudo desenvolvido com a madeira de Astronium sp., Monteiro \& Freitas (1997) encontraram uma perda de massa de $1,85 \%$ em ensaio de apodrecimento acelerado, sob ataque do fungo G. trabeum, perda inferior quando comparadas ao ataque do T. versicolor (Tabela 2). Tal fato se explica pela preferência do fungo G. trabeum a coníferas, sendo chamado de podridão parda, enquanto o fungo $T$. versicolor ataca preferencialmente madeira de folhosas. Araújo et al. (2010) citam a muiracatiara como uma das espécies amazônicas com alta durabilidade natural, capaz de resistir por muitos anos em boas condições de uso em ambientes adversos.

Jesus et al. (1998), avaliando a durabilidade natural da madeira de fava-orelha-de-negro (Enterolobium schomburgkii) em ensaio de campo em condições suscetíveis tanto a fungos quanto a térmitas, observaram que a espécie apresentou alta durabilidade. Tal fato esta em conformidade com Zenid et al. (2009), que mencionam que a madeira de fava-orelha-de-negro é resistente a fungos apodrecedores.

Nota-se que houve menor perda de massa na madeira de A. leiocarpa, a que apresenta densidade aparente (a $15 \%$ de umidade) de $0,83 \mathrm{~g} \mathrm{~cm}^{-3}$, inferior a de $A$. lecointei $\left(0,97 \mathrm{~g} \mathrm{~cm}^{-3}\right)$, e superior a E. schomburgkii $\left(0,79 \mathrm{~g} \mathrm{~cm}^{-3}\right)$, de acordo com o Zenid et al. (2009).

Segundo Marques et al. (2012), o emprego de uma madeira para determinada finalidade pode ser analisado através de sua densidade, uma vez que a sua variação influencia diretamente a durabilidade natural. Panshin \& De Zeeuw (1980) alegam que a densidade, e consequentemente a porosidade, são fatores que influenciam a durabilidade natural. Segundo Bowyer et al. (2003), madeiras mais densas tendem a apresentar teores de extrativos mais elevados e uma maior resistência a organismos xilófagos.

Notou-se ao final do ensaio um leve escurecimento da cor original da madeira das três espécies após o ataque do fungo. Pode-se dizer que a madeira sofre alterações 
em sua cor à medida em que ocorrem reações químicas ou fotoquímicas dos elementos da estrutura anatômica e devido à deterioração provocada por organismos xilófagos (Camargos \& Barros, 2000), ocorrendo alteração nos parâmetros colorimétricos (Almeida et al., 2012).

A porcentagem de perda de massa tem sido o melhor indicador para perda de resistência mecânica, frente a sua classificação de durabilidade natural e de acordo com indicativos de usos de Zenid et al. (2009). Portanto, podem-se considerar as três espécies estudadas com potencial para uso na construção civil em geral, na parte interna, como em vigas, caibros, tesouras, forros e assoalhos; e/ou na parte externa, em que a madeira esteja suscetível ao ataque do fungo apodrecedor T. versicolor, como tábuas, ripas e dormentes.

\section{Conclusões}

A perda de massa registrada pelas madeiras de Astronium lecointei e Enterolobium schomburgkii permitiu classificá-las como resistentes ao fungo Trametes versicolor. A perda de massa da madeira de Apuleia leiocarpa permitiu classificá-la como altamente resistente ao fungo T. versicolor.

Considerando-se a porcentagem de perda de massa das três espécies, pode-se inferir que a madeira de $A$. leiocarpa apresenta menor perda de suas propriedades originais quando utilizada em situações de risco de ocorrência de degradação pelo fungo T. versicolor.

\section{Referências}

Almeida, N. A. et al. Biodeterioração de produtos à base da madeira de cedro australiano (Toona ciliata M. Roem. var. australis). Cerne, v. 18, n. 1, p. 17-26, 2012. DOI: 10.1590/S0104-77602012000100003.

Araújo, B. H. J. et al. Durabilidade de estacas de eucalipto (Eucalyptus sp.) tratadas com CCA após 60 meses de ensaio no campo experimental da Embrapa Acre. Rio Branco, AC: Embrapa Acre, 2010. 8 p. (Embrapa Acre. Comunicado técnico, 176).

Archibald, F. S. \& Addleman, K. Kraft pulp bleaching and delignification by Trametes versicolor. Journal of Biotechnology, v. 53, p. 215-236, 1997.

American Society for Testing and Materials. ASTM D 2017: standard method for accelerated laboratory test of natural decay resistance for woods. West Conshohocken, 2005.
American Society for Testing and Materials. ASTM D 1413: standard test method for wood preservatives by laboratory soil-block cultures. West Conshohocken, 1999.

Bowyer, J. L. et al. Forest products and wood science: an introduction. 4th ed. Ames, Iowa: Iowa State University Press; Oxford: Blackwell, 2003. p. 232-250.

Brasil. Ministério do Meio Ambiente. Plano nacional de silvicultura com espécies nativas e sistemas agroflorestais: PENSAF. Brasília, DF, 2006. 38 p.

Camargos, J. A. A. \& Barros, M. G. Colorimetria e desenho de madeiras de Lyptus. Brasília, DF: Fundação de Tecnologia Florestal e Geoprocessamento, 2000. p. 509-517. Relatório final Aracruz Celulose.

Castellani, A. The "water cultivation" of pathogenic fungi. The Journal of Tropical Medicine and Hygiene, v. 44, p. 217-220, 1964.

FAO. Global forest resources assessment. Rome, 2015. 56 p.

Fosco Mucci, E. S. C. et al. Durabilidade natural de madeiras em contato com o solo IV. Revista do Instituto Florestal, v. 4, n. 2, p. 558-563, 1992.

IBAMA. Madeiras tropicais brasileiras. Brasília, DF, 1997. 152 p.

Jesus, M. A. et al. Durabilidade natural de 46 espécies de madeira amazônica em contato com o solo em ambiente florestal. Scientia Forestalis, n. 54, p. 81-92, 1998.

Marques, S. S. et al. Estudo comparativo da massa específica aparente e retratibilidade da madeira de pau-brasil (Caesalpinia echinata Lam.) nativa e de reflorestamento. Revista Árvore, v. 36, n. 2, p. 373-380, 2012.

Mendes, A. S. \& Alves, M. V. S. A degradação da madeira e sua preservação. Brasília, DF: IBDF/DPq-LPF, 1988. 57 p.

Monteiro, M. B. B. \& Freitas, A. R. Método de ensaio acelerado para avaliação da durabilidade natural de madeiras. Revista Árvore, v. 21, n. 4, p. 555-561, 1997.

Morais, S. A. L. et al. Análise da madeira de Pinus oocarpa: Parte I: estudo dos constituintes macromoleculares e extrativos voláteis. Revista Árvore, v. 29, n. 3, p. 461-470, 2005.

Panshin, A. J. \& De Zeeuw, C. Textbook of wood technology. 4th ed. New York: McGraw Hil, 1980. 722 p.

Tanaka, H. et al. Hydroxyl radical generation by an extracelular low molecular weight substance and phenol oxidase activity during wood degradation by the white rot basidiomycete Trametes versicolor. Journal of Biotechnology. v. 75, n. 1, p. 57-70, 1999. DOI: 10.1016/ S0168-1656(99)00138-8.

Trevisan, H. et al. Degradação natural de toras de cinco espécies florestais em dois ambientes. Floresta, v. 28, n. 1, p. 33-41, 2008.

Zenid, G. J. et al. Madeira: uso sustentável na construção civil. 2. ed. São Paulo: Instituto de Pesquisas Tecnológicas, 2009. 103 p. (IPT publicações, 3010). 